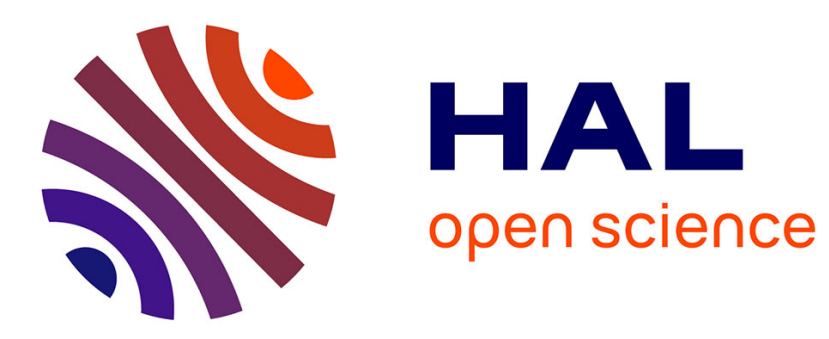

\title{
Lieux de culte à Salamine à l'époque des royaumes
}

Sabine Fourrier

\section{To cite this version:}

Sabine Fourrier. Lieux de culte à Salamine à l'époque des royaumes. Cahiers du Centre d'Etudes Chypriotes, 2015. hal-01450921

\section{HAL Id: hal-01450921 \\ https://hal.science/hal-01450921}

Submitted on 31 Jan 2017

HAL is a multi-disciplinary open access archive for the deposit and dissemination of scientific research documents, whether they are published or not. The documents may come from teaching and research institutions in France or abroad, or from public or private research centers.
L'archive ouverte pluridisciplinaire HAL, est destinée au dépôt et à la diffusion de documents scientifiques de niveau recherche, publiés ou non, émanant des établissements d'enseignement et de recherche français ou étrangers, des laboratoires publics ou privés. 


\section{CENTRE D'ÉTUDES CHYPRIOTES}

CAHIER 45, 2015 
La loi du 11 mars 1957 n'autorisant, aux termes des alinéas 2 et 3 de l'article 41, d'une part, que les « copies ou reproductions strictement privées à l'usage du copiste et non destinées à une utilisation collective » et, d'autre part, que les analyses ou les courtes citations dans un but d'exemple et d'illustration, « toute représentation ou reproduction intégrale, ou partielle, faite sans le consentement de l'auteur ou de ses ayants droit ou ayants cause, est illicite » (alinéa premier de l'article 40).

\section{(C) Centre d'Études Chypriotes, Paris \\ et \\ Édition-Diffusion de Boccard, Paris}

ISSN 0761-8271

ISBN 978-2-7018-0498-9

Illustration de couverture : Métamorphoses de la Grande Déesse, d'après Stella M. Lubsen-Admiraal, dans ce volume, p. 256, fig. 1.

Vignette de titre : Figurine-plaquette Red Polished, d'après Jennifer M. Webb, dans ce volume p. 242, fig. 1. 


\section{CENTRE D'ÉTUDES CHYPRIOTES}

\section{CAHIER \\ 45,2015}

\section{Kypromedousa \\ Hommage à Jacqueline Karageorghis}

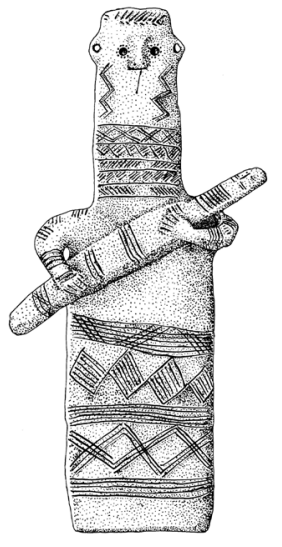

Publié avec le concours de la Fondation A.G. Leventis

Édition-Diffusion De Boccard

11, rue de Médicis, F-75006 Paris 


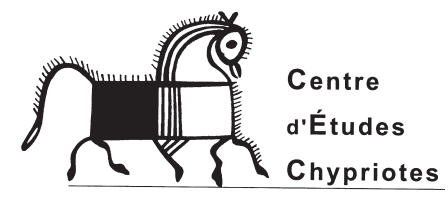

La revue Cahiers du Centre d'Études chypriotes (abrégée CCEC) publie des contributions en allemand, anglais, français, grec, et rend compte d'ouvrages qui lui sont envoyés.

Adresser les propositions d'articles au directeur de la revue (Centre Camille Jullian, Aix).

Directeur de la revue: Antoine HERMARY.

Comité de rédaction : Derek COUNTS, Sabine FOURRIER, Antoine HERMARY, Hartmut MATTHÄUS, Robert MERRILLEES, Marguerite YON, qui constituent aussi le Comité de lecture avec la collaboration de spécialistes extérieurs.

Maquette, mise en page : Marguerite YON.

Centre Camille Jullian, MMSH, Université d'Aix-Marseille - CNRS, 5 rue du Château-de-l'Horloge, B.P. 647, F-13094 Aix-en-Provence Cedex 2. $\quad$ ahermary@mmsh.univ-aix.fr HISOMA [Histoire et Sources des Mondes Anciens], Université Lyon 2-CNRS, Maison de l'Orient, 7 rue Raulin, F-69365 Lyon Cedex 07. sabine.fourrier@mom.fr_marguerite.yon@mom.fr 


\section{SOMMAIRE}

Avant-propos, par Antoine Hermary, Président du Centre 9

In Memoriam : Elisabeth Lagarce (1941-2015), par Annie CAUBET

\section{Dossier «KYPROMEDOUSA » Hommage à Jacqueline Karageorghis}

Introduction : Kypromedousa, «Celle qui règne sur Chypre », par Claire BALANDIER, Brunehilde ImHAus, Eustathios RAPTOU et Marguerite Yon .... 15

Bibliographie de Jacqueline Karageorghis

Vassos Karageorghis, Aspects peu connus de la contribution de Jacqueline Karageorghis à l'archéologie chypriote

\section{Sur les traces de la Déesse}

Robert MerriLleEs, The Mythology Around a Myth:

Aphrodite and Petra tou Romiou

Joanna SMith, Impressing Aphrodite: Stamped Loom Weights from Polis Chrysochous

Thomas KIELy, Finds from Kouklia in the British Museum

Jolanta MŁynarczyk, Moving to a new place: the cult of Paphia Aphrodite in Hellenistic Nea Paphos

Pierre Aupert, Duplex Amathusia

Yannick Vernet, Aphrodite et Apollon.

Essai sur une relation divine privilégiée à Chypre

Christina IoAnnou, D’Aphrodite à Astarté Paphia

Théodoros Mavrojannis, La grotte d'Adonis à Afka au Liban et l'ensemble « grotte-aire sacrificielle » sur l'acropole d'Amathonte à Chypre.

May CHeHAB, L'Aphrodite des Lettres et des Arts chez Marguerite Yourcenar 


\section{Lieux et bâtiments de cultes}

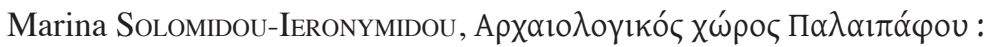

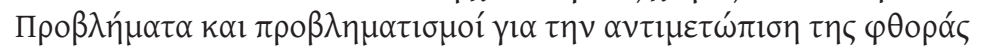
147

Olivier CALlot, À propos du temple du Bronze Récent à Kouklia (Palaipaphos) ..... 157

Claire BALANDIER, Les lieux de culte sur et sous la colline de Fabrika.

Soubassements de temple et autres sanctuaires rupestres

ptolémaïques et romains de Néa Paphos ......................................................... 161

Nota Kourou, Potnia figures and cults in early Iron Age Aegean and Cyprus .......... 181

Anja UlbRich, The Great Goddess at Maroni-Vournes ………………………......... 201

Sabine FourRIER, Lieux de culte à Salamine à l'époque des royaumes ...................... 211

Nancy Serwint, Gifts for the Goddess: Votive Offerings at Ancient Marion ........... 225

\section{Images de la déesse}

Jennifer M. Wевв, The production and distribution of plank-shaped figurines in Middle Bronze Age Cyprus: the Role of Lapithos

Stella M. LubSen-AdmiRaAl, Dromolaxia-Trypes revisited.

Some new thoughts on an old fertility goddess

Annie CAubet et Marguerite Yon, Les multiples visages de la Grande Déesse

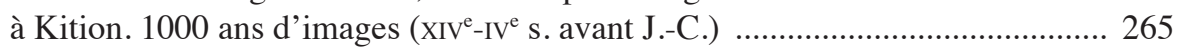

William P. CHILDs, The image of women in the Archaic period ................................... 281

Eustathios RAPTOU, Une tête féminine archaïque en terre cuite de Yialia (district de Paphos)

Evangéline MARKOU, Reconnaître Aphrodite sur les monnaies chypriotes: caractéristiques et particularités locales

Lucie Bonato, De Fénelon à la Grande Déesse de Chypre.

Souvenirs de Léon de Maricourt 1864-1865

Antoine Hermary, Images archaïsantes de l'Aphrodite chypriote ............................. 317

Demetrios Michaelides, Aphrodite at the House of Orpheus in Nea Paphos ............ 329

Cecilia BeER, Baubo amulets around the Mediterranean. Some remarks .................. 339

\section{Chypre hier et aujourd'hui}

Lindy CREWE, An Early-Middle Cypriot Bronze Age zoomorphic figurine from Kissonerga-Skalia

Angelos DelivorRIAS, La règle de la hiérarchie et l'organisation de la composition dans la sculpture monumentale de l'antiquité grecque 363

Pavlos Flourentzos, New Evidence about Theos Hypsistos cult in Roman Cyprus .. 383 
Brunehilde ImHaus, Un exemple de permanence cultuelle de l'Antiquité au Moyen Âge : le monastère de Sainte-Catherine / Saint-Oreste dans le Chrysochou

Joan R. Mertens, Luigi Palma di Cesnola in Libby Prison (1863-1864)

Euphrosyne Rizopoulou-Egoumenidou, La revitalisation des traditions et coutumes à Chypre, contributeurs majeurs du renforcement de l'identité culturelle .....

Yiannis IoANNOU, Multiculturalité à Chypre : réalité historique ou besoin politique .. 417

\section{VARIÉTÉ}

Michel Amandry, Chronique numismatique chypriote (V), 2008-2015

\section{COMPTES RENDUS D'OUVRAGES}

1. Leonardo Fuduli, Fragmenta Paphia. Elementi di decorazione architettonica da Garrison's Camp di Nea-Paphos (Paphia II), Catane, 2015 (M.-Chr. Hellmann)

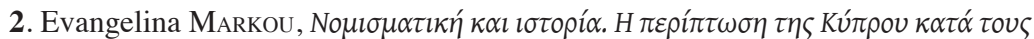

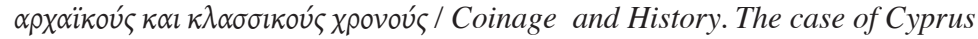
during the Archaic and Classical periods. Nicosie, 2015 (A. Hermary)

3. Luca Bombardieri, Orgoglio e Pregiudizi. L'archeologia cipriota di Luigi Palma di Cesnola alla luce dei documenti e delle corrispondenze con l'Italia, Rome, 2015 (A. Hermary) .. 454

4. Sadie Pickup, Marianne Bergeron, Jennifer M. Webb, Corpus of Cypriote Antiquities 30. Cypriote Antiquities in Reading. The Ure Museum at the University of Reading and the Reading Museum (Reading Borough Council), Uppsala, 2015 (A. Hermary)

5. Stella Demesticha (éd.), Per terram, per mare. Seaborne Trade and the distribution of Roman Amphorae in the Mediterranean, Uppsala, 2015 (A. Marangou)

6. Iosif Hadjikyriakos, Mia Gaia Trentin (éd.), Cypriot Cultural Details.

Proceedings of the 10 $0^{\text {th }}$ Post Graduate Cypriot Archaeology Conference, Oxford, 2015 (A. Caubet, H. Le Meaux) 



\title{
LIEUX DE CULTE À SALAMINE à l'époque des royaumes
}

\section{Sabine FOURRIER}

\begin{abstract}
This article reviews the archaeological evidence attesting the existence of sanctuaries in Iron Age Salamis and in its vicinity. It also addresses some issues concerning the cult as well as the topography of the ancient city and especially of its periurban spaces.
\end{abstract}

Les cultes de Salamine ont été recensés de manière exhaustive par A. Ulbrich ${ }^{1}$ et cette contribution n' apportera rien de neuf, sinon quelques réflexions à propos de la topographie sacrée de la ville et des pratiques qu'elle révèle, en hommage à J. Karageorghis qui, lors du colloque de Lyon de 1978, s’intéressait à la « Grande Déesse » de Salamine 2.

On n'examinera que les vestiges, mobiliers et architecturaux, c'est-à-dire la documentation archéologique, laissant de côté les autres sources ${ }^{3}$. Il faut toutefois en souligner d'emblée la grande disparité. Cette documentation est hétérogène, variable selon les sites et les périodes. On ne possède que rarement de vestiges en place. Dans la plupart des cas, le lieu de culte n'est renseigné que par un matériel décontextualisé, abondant, mais en dépôt secondaire (bothros). Les données sont également de qualités diverses. Les fouilles anciennes sont moins bien documentées que les fouilles récentes. Mais ces dernières, interrompues en 1974, sont, pour certaines, restées inachevées.

Autre contrainte forte, limitant les possibilités d'analyse spatiale, la topographie de Salamine à l'époque des royaumes reste, par ailleurs, mal connue. On n'en saisit que des

1. Ulbrich 2008, p. 417-431.

2. J. Karageorghis, «La Grande Déesse à Salamine », dans Salamine de Chypre. Histoire et archéologie. État des recherches, Paris, 1980, p. 203-213.

3. Notamment littéraires. On pense en particulier au sanctuaire d'Athéna, Agraulos et Diomède mentionné par Porphyre (De Abstinentia II, 54-55 = Salamine X, ${ }^{\circ} 41$ ). L'existence d'un sanctuaire d'Athéna à Salamine est attestée par un décret athénien de l'époque hellénistique (Salamine X, $\mathrm{n}^{\circ}$ 43). Sur le culte d'Agraulos à Salamine, voir M. Valdés Guía, « The Cult of Aglauros (and Aphrodite) in Athens and in Salamis of Cyprus: Reflections on the origin of the Genos of the Salaminioi », Ancient West \& East 4-1, 2005, p. 57-76. 
membres épars : tombes, portion de rempart, établissement comprenant un sanctuaire ${ }^{4}$. Faute de connaître les limites de la ville, qui ont au demeurant varié au cours du temps, certainement même au cours de l'Âge du Fer, il me paraît difficile de distinguer entre sanctuaires urbains, suburbains et périurbains. Je m'intéresserai donc ici à tous les lieux de culte attestés à Salamine et dans son périmètre proche, jusqu'à Enkomi $(F i g .1)^{5}$.

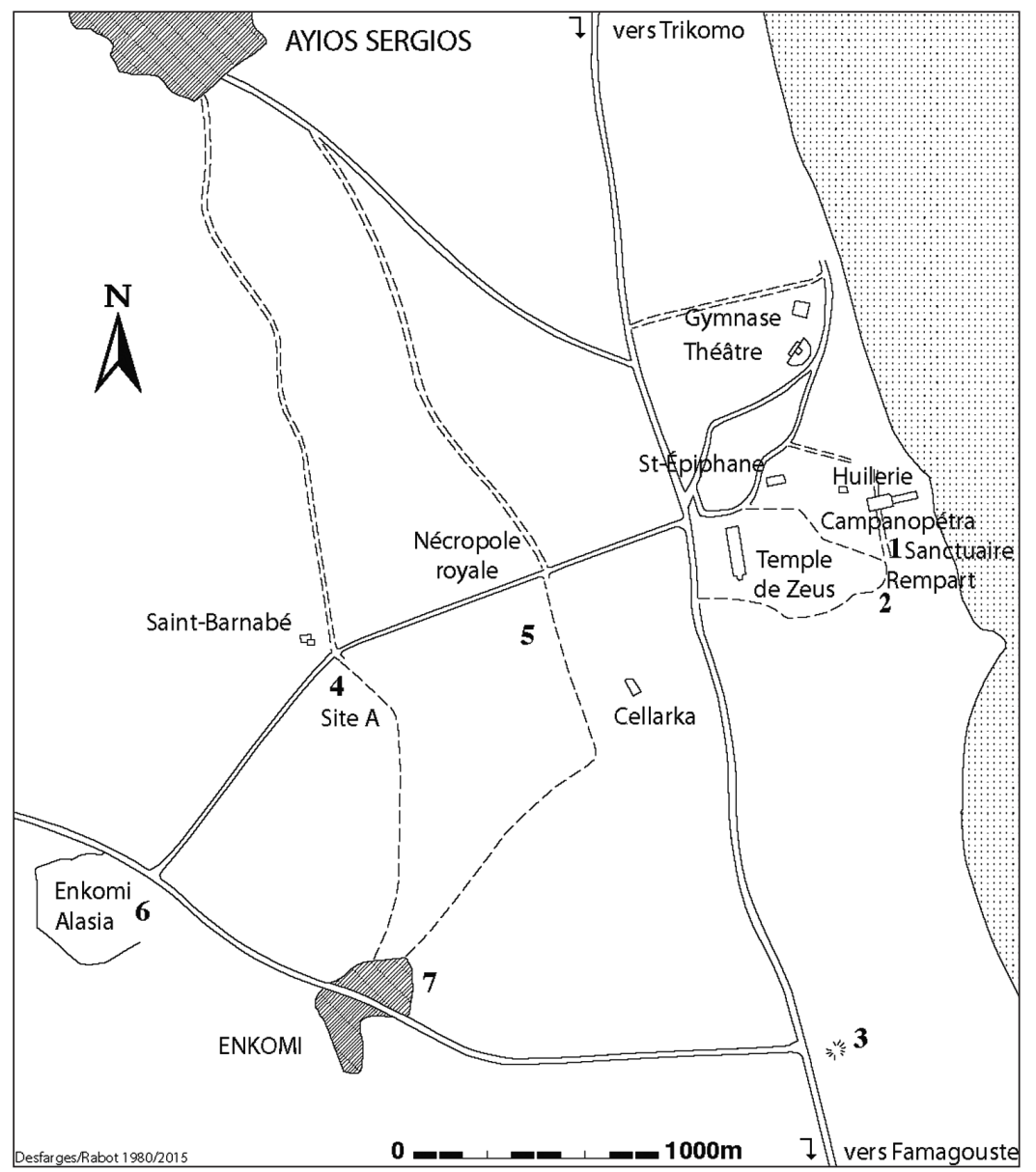

Figure 1. Carte de Salamine.

Les numéros correspondent aux sanctuaires mentionnés dans le texte

(DAO A. Rabot, d'après Paul-A. Pironin 1966, HiSoMA/MOM).

\section{Yon 1993.}

5. Je laisse de côté le sanctuaire mentionné par A. di Cesnola, dont l'existence même paraît douteuse (= Ulbrich 2008, $\mathrm{n}^{\circ}$ SA 8), ainsi que celui du lieu-dit Krines ou Krina, plus éloigné (= Ulbrich $2008, n^{\circ}$ SA 9). 


\section{Le sanctuaire de la Campanopétra (= Ulbrich $2008, \mathrm{n}^{\circ} \mathrm{SA} 1$ )}

C'est le seul site pour lequel on possède des restes construits, documentant une succession de phases d'utilisation, une stratigraphie. C'est aussi le seul lieu de culte dont on puisse affirmer qu'il s'agit d'un sanctuaire urbain, c'est-à-dire localisé intra-muros, puisqu'il s'appuie sur une muraille dont il est séparé par une rue et dont il est, au cours des différentes phases de son existence, contemporain ${ }^{6}$.

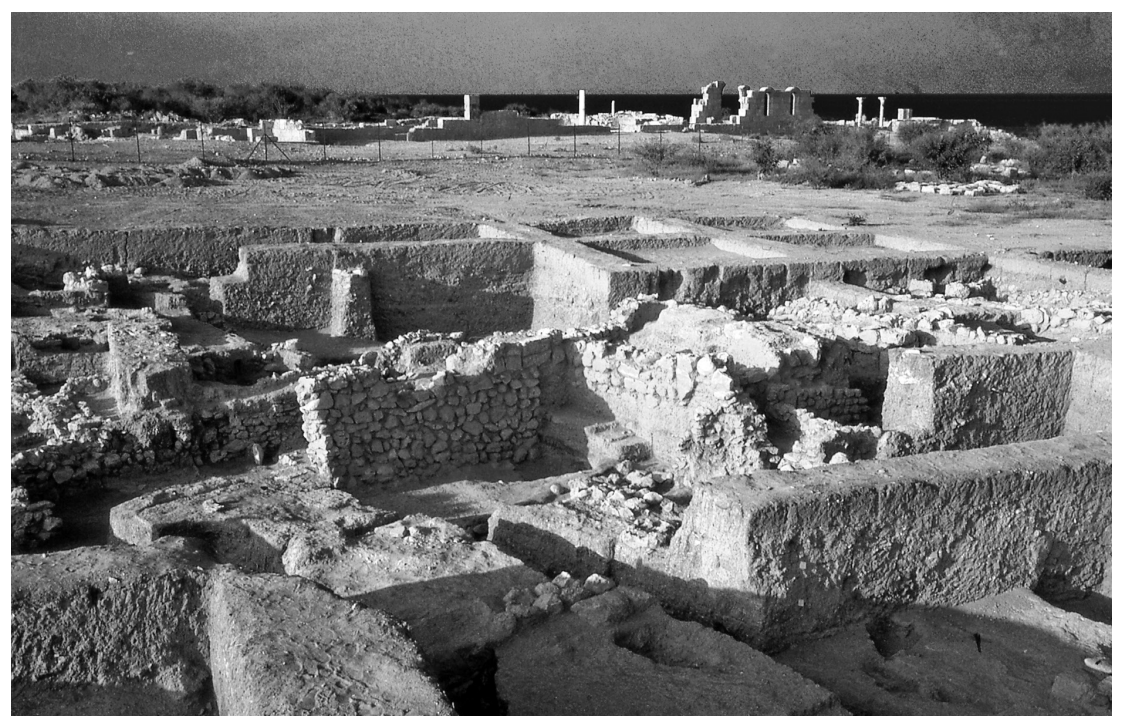

Figure 2. Vue du sanctuaire de la Campanopétra depuis le sud, état 1972. À l'arrière-plan, le complexe basilical.

(Mission de Kition et Salamine).

La fouille n'a pas été achevée et les limites de l'ensemble, comme le phasage des vestiges dégagés dans les différents carrés sont encore difficiles à cerner (Fig. 2). Des sondages profonds, contre le rempart au sud-ouest (sondages $\mathrm{Z}$ et $\mathrm{D}$ ), sous la basilique paléochrétienne au nord et au nord-est, au pied de la résidence byzantine dite de «L'huilerie » au nord-ouest, ont ouvert des fenêtres ponctuelles dans l'épaisseur chronologique de l'occupation du plateau. La stratigraphie y est, autant qu'on puisse en juger, similaire à celle qui a été exposée, de manière plus extensive, dans la zone du sanctuaire. Ce dernier appartenait donc à un ensemble plus vaste, fait de bâtiments implantés selon une orientation constante, en place dès les niveaux les plus anciens.

On peut, d'après les assemblages recueillis, scander l'occupation du sanctuaire (et du plateau) en trois grandes phases ${ }^{7}$.

6. Yon 1993, p. 144 ; Yon 2009, p. 298. Mon exposé s'appuie également sur les archives inédites de la mission, en particulier les carnets de fouille.

7. Les datations, qui reposent sur les notations des carnets et quelques photographies de matériel choisi, sont forcément approximatives. 
La première est géométrique (vers 1050-850 av. J.-C. ?). Elle comprend plusieurs niveaux de sol, dont le plus ancien repose sur le rocher.

La deuxième couvre la fin de l'époque géométrique et le tout début de l'époque archaïque (vers 850-750 av. J.-C. ?). Elle s'achève par une destruction violente : une épaisse couche d'incendie sépare partout les niveaux géométriques des niveaux postérieurs archaïques. Un autel construit en briques est attribuable à cette phase que documente une grande quantité de mobilier, de nature votive, mais aussi technique : bassins de pierre et de terre cuite, déchets métallurgiques signalant, dans ce sanctuaire de Salamine comme dans d'autres sanctuaires contemporains de Chypre ${ }^{8}$, la présence d'ateliers.

La dernière phase est archaïque. Elle comprend au moins trois sols successifs, avant l'abandon (sans destruction violente) du lieu de culte, vers la fin $\mathrm{du}_{\mathrm{VI}}^{\mathrm{e}}$ ou le début du ve s. av. J.-C.

Outre les aménagements (autels, foyers), le riche matériel recueilli, céramique et coroplastique, documente l'existence d'un culte rendu à une divinité probablement masculine au vu des représentations figurées ${ }^{9}$. M. Yon propose d'y voir le premier sanctuaire du grand dieu de Salamine, ensuite appelé Zeus, sanctuaire qui aurait été déplacé à l'époque classique (emplacement inconnu) puis hellénistique (temple de Zeus à l'ouest de la ville) ${ }^{10}$.

\section{La zone au sud du rempart (= Ulbrich $\left.2008, \mathrm{n}^{\circ} \mathrm{SA} 2\right)$}

Les découvertes effectuées au sud du rempart documentent l'existence d'un ou de plusieurs sanctuaires, mais elles ne permettent pas de le(s) localiser précisément (intra- ou extra-muros ?) : le matériel retrouvé n'est pas dans son contexte original d'exposition, il est jeté, c'est un dépôt secondaire.

Le site avait déjà été exploré en 1890 par les archéologues britanniques Munro et Tubbs, qui avaient fait pratiquer un certain nombre de tranchées ${ }^{11}$. Le contexte est complexe, avec à la fois des dépôts anciens et des creusements plus récents qui ont perturbé la stratigraphie. Parmi les figurines de terre cuite archaïques, les fouilleurs signalent des représentations de guerriers, des groupes en char, des chevaux et des ânes

8. Ainsi dans les sanctuaires de Kition, à Kathari et Bamboula. Pour ce dernier site, voir A. Caubet, S. Fourrier, M. Yon, Kition-Bamboula VI. Le sanctuaire sous la colline (TMO 67), Lyon, 2015, p. 97-108.

9. Les figurines de terre cuite sont publiées par T. Monloup, Salamine de Chypre XII. Les figurines de terre cuite de tradition archaïque, Paris, 1984.

10. Yon 1993, p. 146 ; ead. 2009, p. 298-305. Sur la divinité : M. Yon, «Zeus de Salamine», dans R. Bloch (éd.), Recherches sur les religions de l'Antiquité classique, Genève et Paris, 1980, p. $85-103$.

11. Munro, Tubbs 1891, p. 137-145, site « D ». Les fouilleurs britanniques mentionnent sous la même lettre de « very slight operations » sur le site du Daimonostasion, qui est toutefois bien distinct, marquant l'un des points les plus hauts du plateau, au sud-ouest du rempart. Pour ces travaux britanniques, voir également Wilson 1980, p. 62-65. 
bâtés, des protomés de taureaux ; parmi les statuettes de calcaire, des faucons ; parmi les figurines de terre cuite classiques, des représentations féminines à calathos.

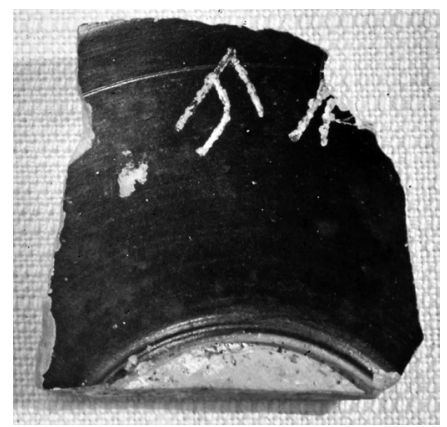

Figure. 3. Fragment de vase attique à inscription syllabique, inv. Sal. 4933

(Mission de Kition et Salamine).
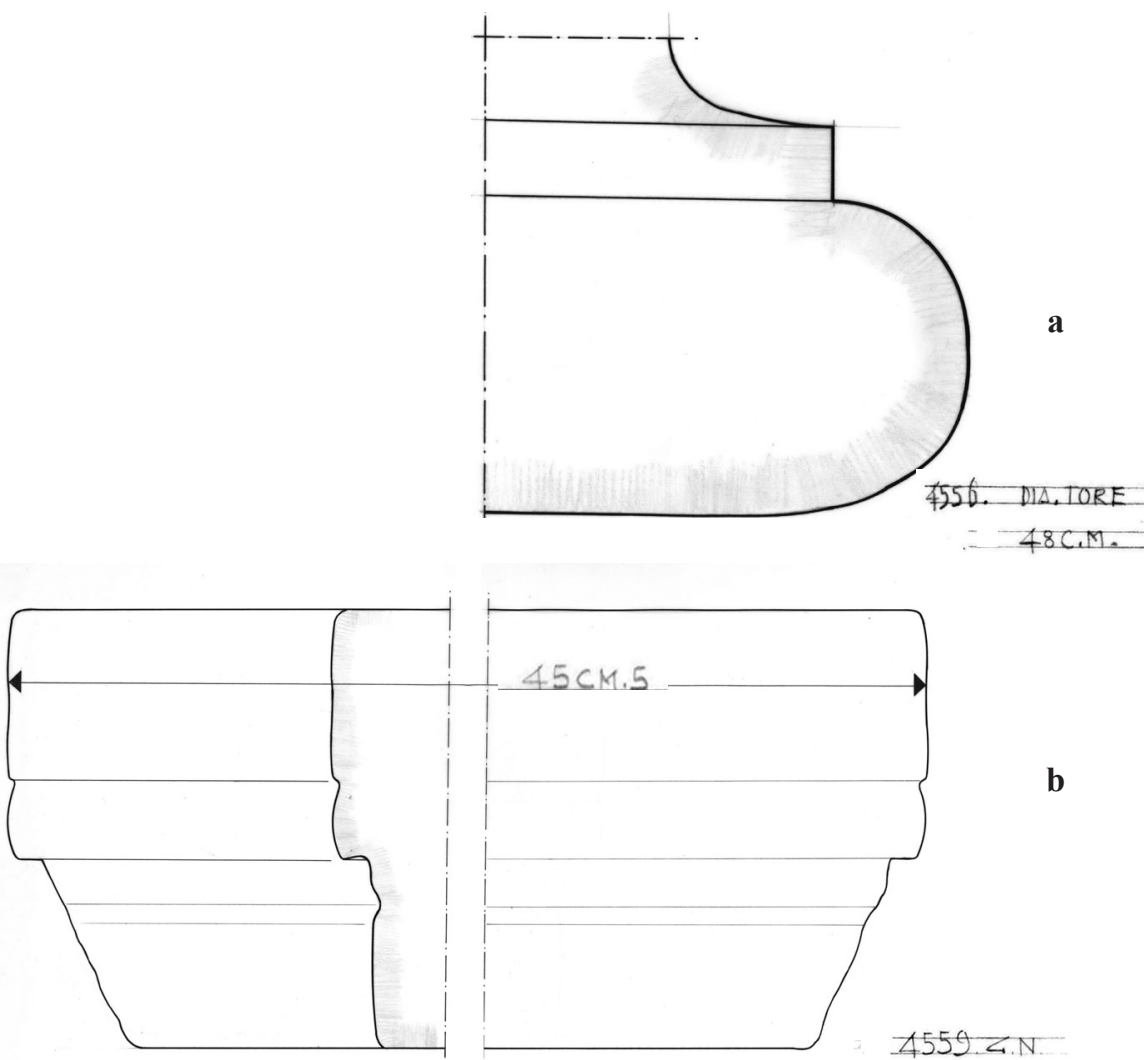

Figure 4. Fragments architecturaux en calcaire.

a. Base de colonne ionique, inv. Sal. $4556, \varnothing 48 \mathrm{~cm}$.

b. Corniche ionique, inv. Sal. 4559, ø $45,5 \mathrm{~cm}$.

(Dessins Paul-A. Pironin, archives Mission de Kition et Salamine). 
Les fouilles françaises ont dégagé au même endroit deux dépôts principaux, l'un apparemment constitué au cours du vi ${ }^{\mathrm{e}} \mathrm{s}$. av. J.-C., l'autre fermé avant le milieu du $\mathrm{II}^{\mathrm{e}} \mathrm{s} .^{12}$ Le matériel votif est extrêmement abondant, en particulier les figurines de terre cuite. Elles semblent surtout représenter des types masculins à l'époque archaïque, des types féminins à l'époque classique ${ }^{13}$. Mais un culte masculin est également attesté parmi le matériel d'époque classique : les fouilleurs britanniques ont découvert plusieurs dédicaces à Zeus Sôter, incisées après cuisson sur des fragments de vases attiques à vernis noir. C'est au même endroit que les fouilles françaises ont mis au jour deux fragments de coupes à vernis noir portant un graffite syllabique $t i$-wo (Fig. 3), peut-être le nom de Zeus au génitif ${ }^{14}$. Autre lot de trouvaille important des fouilles françaises : des fragments architecturaux en calcaire (chapiteau d'ante, fragment de chapiteau ionique, fragments de colonnes, fragment de corniche ionique), qui indiquent l'existence, à proximité, d'un édifice de type grec ionique, datable de la fin du $\mathrm{IV}^{\mathrm{e}} \mathrm{s}$. ou de la première moitié du $\mathrm{III}^{\mathrm{e}} \mathrm{s}$. av. J.-C. ${ }^{15}$ (Fig. 4). Rien n'indique que ce bâtiment ait été érigé dans un sanctuaire, mais l'hypothèse mérite d'être proposée.

\section{Toumpa (= Ulbrich 2008, $\mathrm{n}^{\circ} \mathrm{SA} 5$ )}

Le sanctuaire est certainement périurbain, séparé de Salamine par l'embouchure du Pédiéos. Sa localisation est remarquable, sur une colline rocheuse, qui dominait (surveillait) un accès à la ville depuis le sud dans une zone peut-être lagunaire (accès portuaire ? ${ }^{16}$. Il a été fouillé par la mission britannique de $1890{ }^{17}$. Aucune exploration n'a eu lieu à date postérieure, mais la « Chronique » signale la découverte accidentelle, en 1968, d'un lion fragmentaire en calcaire qui ne paraît pas funéraire ${ }^{18}$.

Les fouilleurs britanniques ne relèvent pas de vestige construit, à l'exception de « some débris ». C'est regrettable, car on ne peut établir si le matériel a été découvert en place ou rejeté dans un bothros. Les découvertes sont de qualité exceptionnelle, notamment les

12. C'est ce qu'indique L. Jehasse, Salamine de Chypre VIII. La céramique à vernis noir du rempart méridional, Paris, 1978, p. 3. La distinction entre les deux dépôts et leur datation sont toutefois mal établies : cf. Salamine XIV, p. 12-14.

\section{Salamine XIV.}

14. Voir le commentaire prudent d'O. Masson reproduit dans J. Pouilloux, P. Roesch, J. MarcilletJaubert, Salamine de Chypre XIII. Testimonia Salaminia 2, n 20. Munro et Tubbs (1891, p. 144) mentionnent également la découverte de graffites en «native script».

15. Découverte mentionnée par J. Pouilloux dans la «Chronique », BCH 93, 1969, p. 542-543.

16. La route conduisant de Famagouste à Salamine traversait encore en 1896, par le biais d'un viaduc, une zone marécageuse : Murray, Smith, Walters 1900, p. 1. Sur la confusion à ne pas faire entre Toumpa et Toumpa tou Michali, qui est un autre toponyme distinct de la région de Salamine (il apparaît sur le relevé des Britanniques, qui y ont fait des sondages : Munro, Tubbs 1891,p. 104106), voir Ulbrich 2008, p. 427, n. 61.

17. Munro, Tubbs 1891, p. 146-166. Voir également Wilson 1980, p. 61-62, qui considère que le site « is evidently a bothros containing debris from a nearby open sanctuary ».

18. BCH 93, 1969, p. 491-493. 
statues moulées de terre cuite, certaines de taille naturelle, voire supérieure ${ }^{19}$. La majorité paraît représenter des types masculins, mais Munro et Tubbs signalent également la découverte de statuettes féminines, de petites dimensions, dans une zone apparemment distincte de celle qui a livré le gros des trouvailles ${ }^{20}$.

La qualité des offrandes distingue assurément le sanctuaire de Toumpa comme un lieu de culte fréquenté par l'élite et consacré à une divinité masculine. Autant qu'on puisse en juger, le matériel découvert date de la période archaïque ( $\mathrm{VII}^{\mathrm{e}}-\mathrm{VI}^{\mathrm{e}} \mathrm{s}$. av. J.-C.) et le site paraît abandonné vers le tout début $\mathrm{du} \mathrm{v}^{\mathrm{e}} \mathrm{s}$. Cet abandon fait écho à celui du sanctuaire de la Campanopétra $\left(n^{\circ} 1\right)$. On peut se demander s'il n'existait pas un rapport étroit de correspondance, mais aussi de différence (dans les modalités du culte et la nature des offrandes consacrées) entre le sanctuaire urbain et le sanctuaire périurbain, peut-être tous deux consacrés à la même divinité.

\section{Saint-Barnabé (= Ulbrich $2008, \mathrm{n}^{\circ} \mathrm{SA} 4$ )}

La région de Saint-Barnabé est séparée de la ville antique par des nécropoles. La «Chronique » signale l'existence de deux sanctuaires, repérés grâce à des découvertes de surface, à proximité du monastère ${ }^{21}$. L'un d'entre eux (« site A »), où avait été recueillie une belle tête de calcaire à calathos végétal, de dimensions naturelles, a été fouillé par M. Calvet (-Yon), mission française, pour le Département des Antiquités en $1968{ }^{22}$. Les tranchées ouvertes n'ont pas mis au jour de vestige construit, à l'exception du dromos creusé dans le rocher d'une tombe plus récente, vraisemblablement hellénistique, qui indique l'extension de la nécropole vers l'ouest ${ }^{23}$. La concentration des offrandes sur une trentaine de mètres carrés définit l'étendue du bothros, sans qu'on ait d'indice sur la localisation du sanctuaire lui-même ${ }^{24}$.

Les trouvailles comprennent surtout des statuettes de calcaire féminines, datables de la fin de la période archaïque et du début de la période classique ( $\mathrm{vI}^{\mathrm{e}}-\mathrm{v}^{\mathrm{e}} \mathrm{s}$. av. J.-C.). Elles attestent un culte rendu à une divinité féminine, peut-être la même que celle à qui étaient offertes les figurines, pour l'essentiel plus récentes et de terre cuite, découvertes au sud du rempart $\left(\mathrm{n}^{\circ} 2\right)$. On peut se demander, comme à propos du sanctuaire de la Campanopétra

19. Les autres découvertes comprennent de nombreuses amulettes de faïence, des scarabées, des lampes, des statuettes de calcaire (humaines et animales), de la céramique, des coquillages...

20. Munro, Tubbs 1891, p. 146 et 158.

21. ВСH 87,1963 , p. 353.

22. $B C H$ 89, 1964, p. 332-333; Salamine V.

23. La tombe publiée la plus occidentale de Salamine est la tombe 80 , située au sud-ouest de la nécropole «royale », au lieu-dit Koufomeron. Elle se trouve à l'est du chemin menant du monastère de Saint-Barnabé au village d'Enkomi. Elle se distingue des autres tombes construites par son architecture et son décor peint. Son fouilleur la date de la fin du vi -début du ve s. av. J.-C. Il signale la découverte alentour de tombes creusées et de pyrés semblables à ceux mis au jour dans la nécropole voisine de Cellarka: Karageorghis 1973, p. 123-127.

24. Salamine V, p. 1-9. 
et de celui de Toumpa, s'il n'y avait pas un rapport d'écho, de complémentarité, entre le sanctuaire (probablement) urbain et le sanctuaire périurbain.

La date de constitution du dépôt marque un moment de réfection ou d'abandon, en tout cas une transformation importante du lieu de culte dans lequel ces offrandes étaient originellement consacrées ${ }^{25}$.

\section{Région de la tombe de Sainte-Catherine (= Ulbrich 2008, $\mathrm{n}^{\circ} \mathrm{SA} 3$ )}

La tombe ou «prison de Sainte-Catherine » fait partie des tombes construites de la nécropole « royale ${ }^{26}$. M. Ohnefalsch-Richter décrit, dans un article de 1883, la « tombe de Sainte-Catherine » au sud de laquelle il aurait trouvé des fragments de statuettes féminines ${ }^{27}$. Dans son répertoire des lieux de culte chypriotes, paru dix ans plus tard, il note que ce sanctuaire, qu'il a fouillé en 1879-1880, est situé à environ 50-80 mètres au sud-est du « grand tumulus de la plaine de Salamine » ${ }^{28}$. Cette expression ne peut désigner que l'imposant tumulus de la tombe 3, exploré par une mission britannique en 1896 et fouillé ensuite par V. Karageorghis ${ }^{29}$. C'est ce que reprend S. Reinach dans sa «Chronique d'Orient » en localisant le sanctuaire « au sud du tumulus d'HagiaKatharina ${ }^{30}$. Les fouilleurs britanniques de 1896 désignent d'ailleurs la tombe 3 sous le nom de «tumulus d'Hagia Caterina ». C'est par rapport à ce tumulus qu'il faut donc situer le sanctuaire d'Ohnefalsch-Richter ${ }^{31}$, dans une zone périurbaine densément occupée par des tombes.

Les trouvailles comprennent quelque 77 fragments de statuettes de terre cuite et de pierre de diverses périodes. Les représentations archaïques et classiques paraissent essentiellement féminines, Ohnefalsch-Richter interprète le lieu de culte comme étant consacré à « Aphrodite-Cybèle ».

25. M. Yon y voit la marque de la politique d'hellénisation d'Évagoras : Salamine V, p. 147-149. A. Ulbrich (2008, p. 427) note la présence d'un fragment de relief daté de la fin du IV s. av. J.-C. mais sa provenance n'est pas assurée : Salamine V, p. 93, n 143.

26. L'édifice originel a été profondément transformé au cours du temps et il était encore utilisé comme chapelle à l'époque contemporaine. Il a été fouillé par V. Karageorghis (1967, p. 90-116).

27. M. Ohnefalsch-Richter, «A pre-historic building at Salamis », JHS 4, 1883, p. 115.

28. M. Ohnefalsch-Richter, Kypros, die Bibel und Homer, Berlin, 1893, p. 13, n 17.

29. Les deux seuls tumulus de la plaine de Salamine sont ceux de la tombe 3 et de la tombe 77 (= « cénotaphe de Nicocréon »), tous deux autrefois datés de l' « âge mycénien » et explorés par la mission britannique de 1896. Pour la tombe 3 : Murray, Smith, Walters 1900, p. 1-3 (et carte en regard de la p. 1 où sont figurés les deux tumulus et où la « tombe de Sainte-Catherine » est symbolisée par un bâtiment surmonté d'une croix, sans tumulus). Publication de la fouille chypriote : Karageorghis 1967 , p. 25-53.

30. S. Reinach, $R A, 1885-2$, p. 350.

31. Contrairement à ce que suggère A. Ulbrich 2008, p. 426, n. 60 . 


\section{Enkomi (= Ulbrich $2008, n^{\circ}$ SA 6 et 7 )}

Le site archéologique d'Enkomi (à ne pas confondre avec le village moderne de même nom) occupe une place particulière dans la topographie salaminienne : c'est la grande ville du Bronze Récent, antécédent immédiat de Salamine. C'est donc un marqueur dans le paysage réel (les vestiges de la ville étaient toujours, du moins en partie, visibles à l'Âge du Fer), mais aussi dans le paysage idéologique (Salamine rattachait ses origines au Nostos de Teucros et donc au monde des héros dont la ville du Bronze Récent portait témoignage).

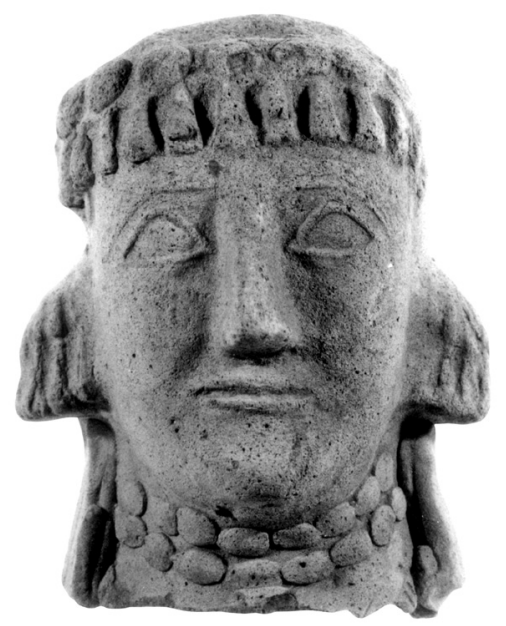

Figure 5. Tête de statuette de terre cuite d'Enkomi, Cyprus Museum inv. $n^{\circ} 1243$ (Department of Antiquities, Cyprus).

Plusieurs sites d'Enkomi entrent dans le paysage sacré de Salamine. Dans la ville du Bronze Récent, du matériel votif de l'Âge du Fer a été retrouvé à la fois en association avec des tombes (tombes 16, 17 et 33 des fouilles britanniques) ${ }^{32}$ et avec la zone du rempart (Area III des fouilles chypriotes sous la direction de P. Dikaios) ${ }^{33}$. Il s'agit de figurines et de statuettes de terre cuite archaïques (Fig. 5), ainsi que de sculptures de calcaire, certaines de grandes dimensions, ce qui prouve, s'il en était besoin, que ces offrandes ne sont pas funéraires, qu'elles ne témoignent pas, pour celles qui ont été retrouvées dans le remplissage du dromos, d'une réutilisation à l'Âge du Fer de tombes du Bronze Récent. L'ensemble a été récemment étudié par A. Hermary qui propose que «les figurines archaïques [des fouilles Dikaios], toutes féminines, proviennent du même

32. Murray, Smith, Walters 1900, p. 26. Les trouvailles conservées au British Museum ont été étudiées par V. Tatton-Brown, «Sculpture and Terracottas from Enkomi in the British Museum », CCEC 30, 2000, p. 17-25.

33. Ce matériel, dont une grande partie était resté inédit, a été publié par M. Hadjicosti 1989. 
lieu de culte que les objets trouvés dans les fouilles anglaises et que l'ensemble a été dispersé à une date qui doit se situer vers la fin de l'époque chypro-archaïque ${ }^{34}$.

On sait, certes, peu de choses du contexte des découvertes anglaises, mais il semble difficile de supposer que le matériel d'un seul et même sanctuaire ait été dispersé, notamment dans le remplissage du dromos de tombes. La concentration des trouvailles dans la zone du rempart peut sans doute indiquer l'existence d'un sanctuaire (dont on n'a pas retrouvé de vestige construit) ${ }^{35}$. D'autres lieux de culte, implantés sur des sites du Bronze Récent, sont attestés dans l'île, et c'est même l'un des traits saillants de la phase de «territorialisation » des royaumes de Chypre au cours de l'époque archaïque ${ }^{36}$. Une autre hypothèse est cependant possible, qui rendrait mieux compte des trouvailles des tombes : on pourrait supposer l'existence non pas d'un lieu de culte (dont le matériel aurait été dispersé), mais de cérémonies, ponctuelles ou répétées, qui conduisaient les gens de Salamine, à certaines occasions, sur les ruines d'Enkomi ${ }^{37}$. Les trouvailles correspondraient alors aux offrandes qui étaient déposées dans ou auprès de certains marqueurs topographiques du site : tombes, rempart.

\section{7. « Tombe $77 »$}

Avant de clore ce panorama, il faut mentionner un dernier lot de trouvailles, provenant cette fois non pas de la ville du Bronze Récent d'Enkomi, mais des limites du village moderne d'Enkomi. On a déjà mentionné le grand tumulus qui, avec celui de la tombe 3 (dit « tumulus de Sainte-Catherine » dans les rapports anciens), marquait le paysage aux abords sud-ouest de Salamine. Exploré par la mission britannique de 1896, il a été fouillé par V. Karageorghis ${ }^{38}$.

34. A. Hermary, « Les cultes à Chypre, du Bronze Récent au I ${ }^{\text {er }}$ millénaire : permanence des sanctuaires et nouveaux 'lieux de mémoire' », dans G. Cadogan, M. Iacovou, K. Kopaka, J. Whitley (éd.), Parallel Lives: Ancient Island Societies in Crete and Cyprus (British School at Athens Studies 20), Londres, 2012, p. 280-288 (citation p. 283).

35. M. Hadjicosti (1989, p. 117) associe ce sanctuaire à un habitat, documenté par une nécropole (de la fin de l'époque géométrique et du début de l'époque archaïque) au lieu-dit Kaminia, à environ $900 \mathrm{~m}$ au nord du site d'Enkomi. On peut toutefois se demander si ces tombes, certes relativement éloignées, ne sont pas liées à l'occupation de la ville de Salamine.

36. Hadjicosti 1989, p. 117-119 ; S. Fourrier, La coroplastie chypriote archaïque (TMO 46), Lyon, 2007, p. 122 ; G. Papantoniou, « Cypriot sanctuaries and religion in the Early Iron Age: Views from before and after », dans M. Iacovou (éd.), Cyprus and the Aegean in the Early Iron Age. The Legacy of Nicolas Coldstream, Nicosie, 2012, p. 285-319 (en particulier p. 297-301).

37. L'idée était déjà suggérée par P. Dikaios, Enkomi I, Mayence, 1969, p. 302 : « the memory of the town and in particular of the worship in it of various deities, attracted people who deposited various offerings as pottery or terracotta figurines ». Peut-être ces marques de dévotion individuelles prenaient-elles place dans le cadre de cérémonies officielles, encouragées ou organisées par le pouvoir royal.

38. Karageorghis 1973, p. 128-202. 
Je ne reviens pas sur les différentes interprétations qui ont été proposées de ce monument exceptionnel (qui n'est pas une tombe) et qui lui prêtent un caractère soit funéraire ${ }^{39}$, soit cultuel ${ }^{40}$. Du comblement du tumulus proviennent quatre statues de calcaire fragmentaires et des terres cuites archaïques que le fouilleur attribue à un sanctuaire plus ancien, localisé à proximité ${ }^{41}$. On ne peut guère en dire plus.

Ce tableau, forcément fragmentaire, ne peut suffire à rendre compte du paysage sacré de Salamine à l'Âge du Fer. On se contentera de quelques remarques, de quelques pistes de réflexion.

Seul sanctuaire sûrement urbain, celui fouillé par la mission française au sud de la basilique $\left(n^{\circ} 1\right)$ était localisé près d'un rempart qui ceignait la ville ou, peut-être, le seul plateau de la Campanopétra (auquel ne se limitait pas forcément la ville de Salamine aux époques géométriques et archaïques). Ses niveaux construits les plus anciens, qui reposent sur le rocher, datent du début de l'époque géométrique : c'est ce que montre la découverte, dans les couches les plus profondes, de céramique Proto-White Painted. C'est donc le seul lieu de culte urbain de Chypre à être fondé au début de l'Âge du Fer (sans antécédent du Bronze Récent) et à être fréquenté sans solution de continuité entre le $\mathrm{XI}^{\mathrm{e}}$ et la fin du $\mathrm{VI}^{\mathrm{e}} \mathrm{s}$. av. J.-C. Sa consécration à une divinité masculine, ensuite honorée sous le nom de Zeus, est probable ${ }^{42}$, mais il est impossible, en l'état de nos connaissances, de savoir s'il s'agissait du seul sanctuaire consacré à cette divinité dans la ville. Il est également difficile d'expliquer les raisons de son abandon, d'autant plus que l'ensemble du plateau, et non pas le seul sanctuaire, semblent à cette époque désertés ${ }^{43}$. Un rapprochement s'impose avec le sanctuaire d'Athéna, sur l'acropole d'Idalion-Ampelliri, abandonné vers la même période ${ }^{44}$. Comme le culte de Zeus à Salamine, le culte d'Athéna à Idalion ne

39. V. Karageorghis propose d'y voir le cénotaphe du dernier roi chypriote de Salamine, Nicocréon.

40. C. Baurain («La contribution des Teucrides aux cultes royaux de l'époque hellénistique », dans P. P. Iossif, A. S. Chankowski, C. C. Lorber [éd.], More than men, less than gods. Studies in royal cult and imperial worship [Studia Hellenistica 51], Louvain, Paris et Walpole, 2011, p. 121-155) suggère que le monument a été érigé lors d'une célébration solennelle en l'honneur des Teucrides. La plateforme et le foyer qu'elle supportait seraient liés à une cérémonie en l'honneur de la dynastie royale salaminienne et de son fondateur Teucros. Le tumulus qui les recouvrait serait postérieur : il aurait été érigé pour recouvrir et protéger les vestiges de cette célébration.

41. Karageorghis 1973, p. 191-192.

42. Yon 2009, p. 298.

43. Voir les remarques de J. Pouilloux dans la «Chronique », BCH 95, 1971, p. 398.

44. E. Gjerstad, J. Lindros, E. Sjöqvist, A. Westholm, The Swedish Cyprus Expedition. Finds and Results of the Excavations in Cyprus II, Stockholm, 1935, p. 460-628. Les Suédois lient l'abandon du sanctuaire à la conquête de la ville par Kition (ibid., p. 628), ce qui les oblige à privilégier une date haute pour la fin du royaume indépendant, que contredisent les données numismatiques : voir l'exposé d'A. Georgiadou, « La tablette d'Idalion réexaminée », CCEC 40, 2010, p. 141-203 (en particulier p. 162-170). 
s'arrête pas avec l'abandon du sanctuaire : une inscription royale postérieure prouve que le culte de la déesse a été entretenu par les nouveaux maîtres phéniciens de la ville ${ }^{45}$. Il faut donc supposer que, comme Zeus à Salamine, Athéna a « déménagé » ${ }^{46}$ à Idalion.

La découverte de dépôts votifs documente, sinon l'abandon, du moins le réaménagement de lieux de culte. Le sanctuaire périurbain de Toumpa $\left(\mathrm{n}^{\circ} 3\right)$ cesse d'être fréquenté à peu près en même temps que le sanctuaire urbain de la Campanopétra. C'est à une date voisine qu'est constitué le premier dépôt au sud du rempart, celui où dominent les ex-voto de type masculin $\left(n^{\circ} 2\right)$. D'autres dépôts à peu près contemporains, découverts dans d'autres villes chypriotes, ont été généralement mis en relation avec les événements de la révolte ionienne : dépôt de la « rampe des Perses » à PalaepaphosMarcello, dépôt du rempart Nord et Terrasse Ouest d'Amathonte ${ }^{47}$. Mais l'explication est difficile pour Salamine, seule capitale de royaume à ne pas avoir été assiégée par les Perses, selon Hérodote (V, 115). On peut noter, de même, la coïncidence entre la date de constitution du second dépôt au sud du rempart $\left(n^{\circ} 2\right)$ et la mise en chantier du grand temple hellénistique de Zeus ${ }^{48}$. Mais il serait hasardeux d'en conclure que ce matériel provient du sanctuaire classique du dieu, qui aurait été alors étroitement associé à une grande déesse. De fait, les représentations figurées d'époque classique sont en grande

45. Dédicace à Anat du roi Baalmilk II, datée de 425 av. J.-C., inscrite sur un bloc architectural de marbre remployé dans l'église de Saint-Georges (le roi avait consacré un monument, $M^{`} Q$, d'interprétation incertaine, à la déesse). Voir M. Yon, Kition-Bamboula, V. Kition dans les textes,

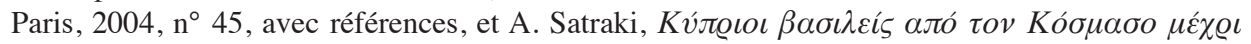

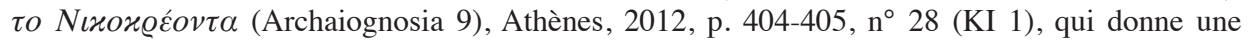
photographie en couleurs de la pierre. Ce bloc est vraisemblablement un épistyle couronnant un petit édifice ou naïskos (peut-être le $M^{\prime} Q$ du texte, qui peut toutefois aussi désigner ce qui était exposé dans l'édifice). On le rapprochera du monument, plus tardif et légèrement plus grand, à inscription bilingue, des hiéronautes de Tyr à Délos, récemment réexaminé par A. Hermary, «Une petite énigme délienne : les “curieuses offrandes" des hiéronautes de Tyr », RA 2014, p. 271-284.

46. Pour reprendre l'expression de M. Yon 2009, p. 302.

47. Palaepaphos-Marcello : voir, en dernier lieu, D. Leibundgut Wieland, «Schändung von geweihten Statuen, Stelen und Steinmalen auf Cypern. Trümmer eines archaischen Heiligtums in der persischen Belagerungsrampe in Alt-Paphos », dans C. Leypold, M. Mohr, C. Russenberger (éd.), Weiter- und Wiederverwendungen von Weihestatuen in griechischen Heiligtümern, Rahdem. Westf., 2014, p. 113-125, pl. 20-22 (je remercie H. Dridi de cette référence). Amathonte, dépôt du rempart Nord : B. Blandin, S. Fourrier, « Le dépôt archaïque du rempart Nord d'Amathonte, I. Introduction : le contexte », BCH 127, 2003, p. 101-105. Amathonte, Terrasse Ouest : A. Hermary, «La "Terrasse Ouest" d'Amathonte (Chypre) : bilan et hypothèses », dans B. Geyer, V. Matoïan, M. Al-Maqdissi (éd.), De l'île d'Aphrodite au paradis perdu, itinéraire d'un gentilhomme lyonnais (Hommage Y. Calvet) (Ras Shamra-Ougarit XXII), Louvain, Paris et Bristol, 2015, p. 29-38 (qui fait le rapprochement avec le dépôt de Salamine).

48. Qui est daté de la fin du II ${ }^{\text {e }}$ s. av. J.-C. : G. Argout [sic, pour Argoud], O. Callot, B. Helly, A.-M. Larribeau, « Le temple de Zeus à Salamine », RDAC 1975, p. 122-141, pl. XVI-XVII. 
majorité féminines. Le monnayage des rois de Salamine rend compte, à partir du IV s., de ce changement iconographique ${ }^{49}$.

La plupart des sanctuaires repérés sont localisés dans l'espace périurbain. Ils dessinent, avec les tombes, un paysage funéraire et sacré qui s'étend aux portes de la ville. Certains lieux de culte périurbains paraissent entretenir un rapport de correspondance, de complémentarité, voire de duplication avec des lieux de culte urbains. On pense encore à Idalion où, d'après la tablette, Athéna possédait au moins deux sanctuaires, l'un sur l'acropole (celui où devait être déposée copie du contrat), l'autre dans la campagne proche, près du terrain octroyé à Onasilos. On manque de sources pour en saisir précisément les rapports. Enkomi, enfin, jouait sans doute un rôle important dans la topographie sacrée de Salamine, au moins à partir de l'époque archaïque. Là encore, il est difficile de comprendre ces rapports, cultuels et mémoriels, avec le passé du royaume, matérialisé par les ruines. Il est toutefois tentant de rattacher ces manifestations, dans le paysage réel, à celles que documentent les textes postérieurs, dans le paysage du mythe ${ }^{50}$.

HiSoMA CNRS, Maison de l'Orient (Université Lyon-2)

\section{BIBLIOGRAPHIE}

Hadicosti (M.), 1989, « More evidence for a Geometric and Archaic rural shrine of a female divinity at Enkomi », RDAC, p. 111120, pl. XXII-XXIII.

KARAGEORGHIS (V.), 1967, Excavations in the Necropolis of Salamis I, Nicosie.

Karageorghis (V.), 1973, Excavations in the necropolis of Salamis III, Nicosie.

Munro (J.A.R.), Tubbs (H.A.), 1891, « Excavations in Cyprus, 1890 », JHS 12, p. 59-198, pl. IV-X.

Murray (A.S.), Smith (A.H.), Walters (H.B.), 1900, Excavations in Cyprus, Londres.

Salamine V : M. Yon, Salamine de Chypre V. Un dépôt de sculptures archaïques, Paris, 1974.

Salamine X : M.-J. Chavane, M. Yon, Salamine de Chypre X. Testimonia Salaminia 1, Paris, 1978.
Salamine XIV : T. Monloup, Salamine de Chypre XIV. Les terres cuites classiques. Un sanctuaire de la Grande Déesse, Paris, 1994.

Ulbrich (A.), 2008, Kypris. Heiligtümer und Kulte weiblicher Gottheiten auf Zypern in der kyproarchaischen und kyproklassischen Epoche (Königszeit), Münster.

Wilson (V.), 1980, " The Tubbs-Munro excavations at Salamis $1890 »$, dans Salamine de Chypre. Histoire et archéologie. État des recherches, Paris, p. 59-70.

Yon (M.), 1993, « La ville de Salamine. Fouilles françaises 1964-1974 », dans M. Yon (dir.), Kinyras. L'archéologie française à Chypre, Lyon, p. 139-158.

YON (M.), 2009, « Le culte impérial à Salamine », CCEC 39, p. 289-308.

49. Avec l'apparition du type d'Aphrodite: E. Markou, Coinage and History. The case of Cyprus during the Archaic and Classical periods, Nicosie, 2015, p. 121-125.

50. P. Christodoulou, « Les mythes fondateurs des royaumes chypriotes. Le nostos de Teukros », CCEC 44, 2014, p. 191-215. 